\title{
Teoria do comportamento planejado - testando a influência da expectativa de desempenho na intenção de jogar on-line
}

\author{
André Luiz da Cunha Silva Mestrando em Administração e Desenvolvimento Empresarial (MADE). Universidade Estácio de Sá (UNESA) - Brasil. \\ cunha.andrel@gmail.com \\ Paulo Roberto da Costa Vieira Doutor em Administração (COPPEAD/UFRJ). Universidade Estácio de Sá (UNESA) - Brasil. \\ paulo.vieira@estacio.br
}

\section{RESUMO}

A demanda por jogos eletrônicos on-line vem crescendo e está projetada mundialmente para atingir US\$118,6 bilhões em 2019. Estudos anteriores analisaram jogos eletrônicos on-line através de modelos de adoção de tecnologia como TPB (Teoria do Comportamento Planejado, do inglês Theory of Planned Behavior) e TAM (Modelo de Aceitação de Tecnologia, do inglês Technology Acceptance Model), mas excluíram o construto expectativa de desempenho por entenderem estar vinculado à eficiência e performance no contexto empresarial. Este estudo tem como objetivo analisar a influência da Expectativa de Desempenho na adoção de tecnologia de jogos on-line considerando o comportamento desse construto também importante quando relacionado ao entretenimento. Esse estudo empírico envolveu 186 participantes que responderam a um questionário estruturado, já testado previamente. Os dados obtidos foram tratados com modelagem de equações estruturais (MEE), utilizando mínimos quadrados parciais (MQP). Como resultado, a hipótese principal foi confirmada, a dimensão "Expectativa de Desempenho" exerceu impacto positivo na intenção de jogar para essa amostra.

Palavras-chave: Jogos On-line, Adoção de Tecnologia, Mínimos Quadrados Parciais.

\section{Theory of planned behavior - testing performance expectation on intention to play on-line games}

\begin{abstract}
On-line games demand has been growing and is projected worldwide to reach $\$ 118.6$ billion in 2019. Earlier studies have analyzed on-line games through technology adoption models such as TPB (Theory of Planned Behavior) and TAM (Technology Acceptance Model), but they excluded performance expectation construct because efficiency and performance are used usually in business context. This study aims to analyze the influence of Performance Expectation in on-line games technology adoption considering this construct also important when related to entertainment. This empirical study involved 186 participants who answered a structured questionnaire, already previously tested, whose main dimension was Experience of Involvement. The obtained data were treated with structural equation modeling (SEM), using partial least squares (PLS). The results confirmed the main hypothesis: Performance Expectation dimension has a positive impact on the intention to play on-line games, for this sample.
\end{abstract}

Keywords: On-line Games. Technology Adoption. Partial Least Squares. 


\section{INTRODUÇÃO}

O mercado de jogos eletrônicos vem crescendo a cada ano e atingirá, em 2019, US\$ 118,6 bilhões. Em 2016, a expectativa era da geração de receitas na ordem de US\$ 99,6 bilhões, um aumento de 8,5\% comparativamente ao ano anterior. O Brasil está em $12^{\circ}$ lugar na lista dos países que mais faturam com jogos eletrônicos, contribuindo com uma receita prevista de US\$ 1,3 bilhões em 2016 (NEWZOO, 2016), o primeiro na América Latina (Tabela 1) - para uma população on-line de 102 milhões de pessoas (PORTAL BRASIL, 2016).

Tabela 1 - Receita de jogos on-line prevista para 2016

\begin{tabular}{c|c|c} 
Participação & País & $\begin{array}{c}\text { Receitas em } \\
\text { Milhões (US\$) }\end{array}$ \\
\hline 1 & Brasil & 1.300 \\
2 & México & 1.100 \\
3 & Argentina & 409 \\
4 & Colômbia & 264 \\
5 & Venezuela & 209 \\
& Outros & 818 \\
\hline & Total & 4.100
\end{tabular}

Fonte: Portal Brasil (2016)

Inicialmente utilizado para entretenimento, os jogos têm servido a outros fins como facilitadores na aprendizagem, em inovações na indústria, como pesquisa de mercado no apoio ao marketing e até como profissão. Jogos e aprendizagem estão se unindo para facilitar o ensino de matemática para crianças nas escolas (KIM; CHANG, 2010). Jogos de empresas na área de logística estão sendo utilizados para testar experimentalmente elementos de pesquisa, confirmando ou refutando teorias existentes (BOUZADA, 2013).

Novos trabalhadores cibernéticos já não procuram empresas tradicionais, e tampouco buscam a esfera pública para trabalhar, utilizando a troca de objetos virtuais e moedas usadas em jogos on-line como atividade econômica no mundo real (LEE; LIN, 2011). O McDonalds, no Japão, investiu US \$2,2 milhões para desenvolver um jogo de treinamento Nintendo DS cuja instalação, em cada um de seus 3.700 estabelecimentos, objetivava aumentar a rentabilidade da rede. Os hotéis da rede Hilton lançaram o Ultimate Team Play, no console Sony PSP, para que seus funcionários completassem tarefas virtuais específicas dentro de um determinado período de tempo. A empresa de tecnologia Cisco utiliza jogos on-line na preparação de suas certificações, onde os jogadores interagem virtualmente se preparando para executar tarefas reais (TAY, 2010).

À medida que o mundo real e os mundos virtuais se interconectam, empresas e pessoas físicas estão se tornando mais experientes nessas tecnologias, cunhando o termo Jogos Sérios para se referir a jogos usados para outros propósitos que não o entretenimento puro (WASKO et al., 2011).

No cenário descrito, avaliar os fatores que influenciam a intenção de jogar torna-se relevante e tem chamado a atenção de pesquisadores. Pesquisas anteriores buscam o melhor modelo para explicar o construto "Intenção de Jogar" a partir de modelos de adoção de tecnologia utilizados quando se deseja estudar esse tema (CHINOMONA, 2013; HO; CHANG; LEE, 2014; HSU; LU, 2004).

Utilizar um modelo de aceitação de tecnologia é buscar identificar o impacto de variáveis em intenções, a partir de construtos que afetam a aceitação de tecnologias (NOBRE; RAMOS; NASCIMENTO, 2011). De acordo com Lee (2009), o modelo de adoção de tecnologia TAM não é um modelo útil para jogos on-line, embora tenha sido amplamente utilizado no estudo da adoção de tecnologias. O pesquisador afirma que o modelo TPB seria mais adequado para explicar e prever o uso de tecnologia orientada ao entretenimento, como jogos on-line, já que une condições facilitadoras à intenção comportamental, influenciando tanto a intenção de jogar quanto o comportamento.

Este estudo se propõe a reaplicar a escala utilizada por Lee (2009) e testar sua relevância estatística no contexto brasileiro adicionando o construto Expectativa de Desempenho.

Venkatesh et al. (2003), na formulação da UTAUT (do inglês: Unified theory of acceptance and use of technology), incluíram o construto Expectativa de Desempenho positivamente relacionado com o uso da tecnologia. 
No modelo adotado nesse estudo também foi incluído esse construto já que a expectativa de ter um bom desempenho no jogo pode se traduzir em um fator motivador que aumente a intenção de jogar.

Considerando que a motivação, seja ela consciente ou inconsciente, está presente no dia a dia e estimula a ação (BAGATTOLI; MÜLLER, 2016), esse estudo se propõe a testar se a expectativa de desempenho serve como fator motivador para a intenção de jogar e a sua relevância no contexto dos jogos eletrônicos on-line.

\section{MODELOS DE ACEITAÇÃO DE TECNOLOGIA}

Considerando jogo on-line como um aspecto da tecnologia da informação orientada para o entretenimento (HSU; LU, 2004), podemos aplicar modelos de aceitação para avaliar jogo on-line assim como já foram utilizados para avaliar adoção de tecnologia frente a aspectos como implementações de sistemas de gestão empresarial (ERP, do inglês: Enterprise Resource Planning), sistemas de inteligência de negócios ( $\mathrm{BI}$, do inglês: Business Intelligence) (PILZ; FERRAZ, 2013) e sistemas de comércio eletrônico (e-Commerce) (XIAO; BENBASAT, 2007).

Dentre as diversas teorias sobre aceitação da tecnologia existentes, tema frequente é a relação entre um conjunto de fatores influenciando a aceitação e a intenção de aceitar ou usar a tecnologia. Nesse estudo, seguiremos o modelo TPB para responder à questão problema, conforme optado por Lee (2009), onde intenção, norma subjetiva e percepção de controle comportamental influenciam a intenção que, por sua vez, influencia no comportamento. A seguir, revisaremos os modelos que influenciaram Lee na sua escolha pela TPB e os que baseiam a inclusão do construto Expectativa de Desempenho no modelo final.

Inicialmente Ajzen e Fishbein, a partir de suas origens na psicologia social, criaram a Teoria da Ação Racional (FISHBEIN; AJZEN, 1975). Esse modelo define as relações entre crenças, atitudes, normas, intenções e comportamentos dos indivíduos, admitindo que o comportamento de um indivíduo é determinado por sua intenção comportamental. Essa intenção, por sua vez, é determinada por atitudes e normas subjetivas de cada pessoa em relação ao comportamento. A Figura 1 ilustra essa teoria, sendo resumida por meio da seguinte equação:

Intenção comportamental $=$ Atitude + Normas subjetivas

Figura 1 - TRA-Teoria da Ação Racional (Theory of Reasoned Action)

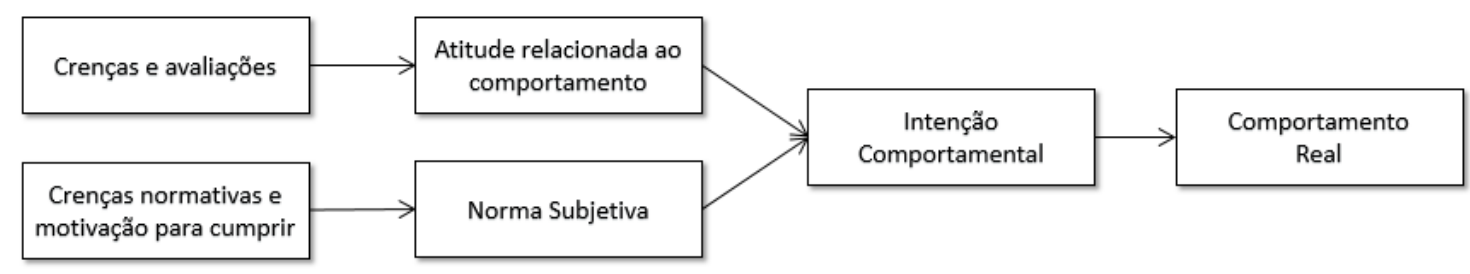

Fonte: Adaptado de Fishbein e Ajzen (1975)

Posteriormente, o próprio Ajzen (1991) evoluiu seu estudo da TRA para a TPB incluindo um terceiro elemento ao modelo anterior, conforme ilustra a Figura 2, gerando o modelo no qual a intenção comportamental só pode encontrar expressão no comportamento se o comportamento em questão estiver sob controle da força de vontade, ou seja, o indivíduo é quem decide executar ou não o comportamento.

Figura 2 - Teoria do Comportamento Planejado (do inglês TPB)

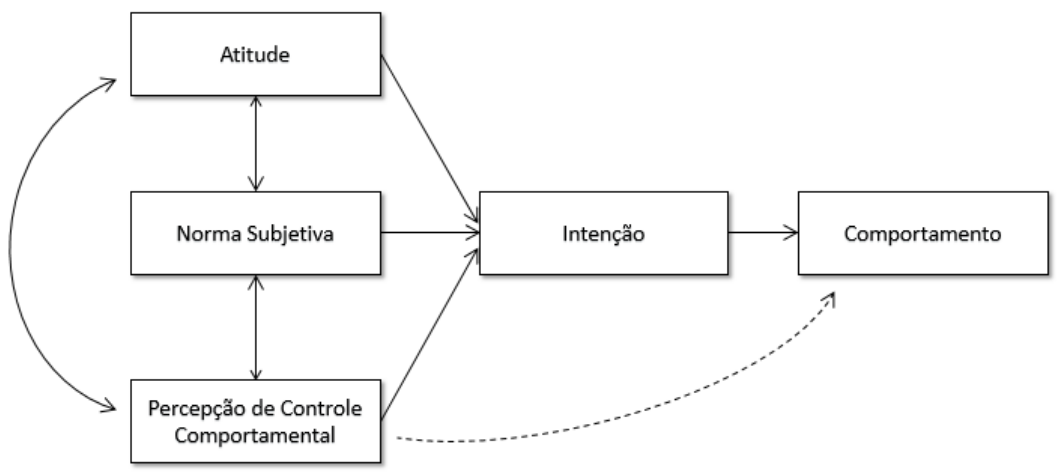

Fonte: Adaptado de Ajzen (1991). 
Como uma evolução do TPB, Davis (1985) propôs o modelo TAM que tornou-se modelo-chave na compreensão de preditores de comportamento humano em relação ao potencial de aceitação ou rejeição de tecnologia (MARANGUNIĆ; GRANIĆ, 2015). O TAM é derivado diretamente do TRA e se baseia em dois determinantes: percepção de utilidade e percepção de facilidade no uso. Davis supôs que esses dois determinantes influenciam diretamente a atitude de uso e que esta, por sua vez, afeta a utilização de sistemas, conforme ilustrado na Figura 3.

Figura 3 - Modelo TAM original proposto por Davis

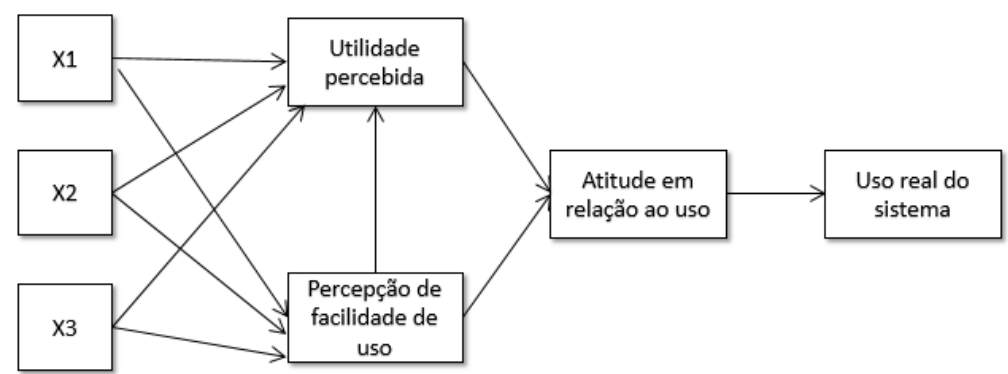

Fonte: Adaptado de DAVIS (1986)

Ao longo dos anos, o modelo TAM foi modificado pelo aludido autor incluindo outros determinantes, conforme sua experimentação prosseguia (DAVIS; BAGOZZl; WARSHAW, 1989). Finalmente, em 2000, foi detalhado o modelo de aceitação TAM que seria aplicado a partir de então, conforme mostra a Figura 4 (VENKATESH; DAVIS, 2000).

Figura 4 - Modelo TAM final

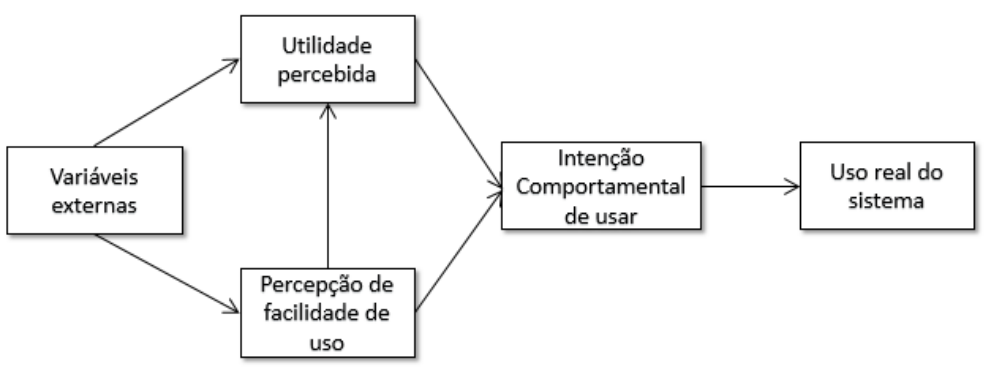

Fonte: Adaptado de Venkatesh e Davis (2000)

Venkatesh seguiu sua pesquisa e junto com Morris, Davis e Davis (VENKATESH et al., 2003) formularam o modelo UTAUT, reunindo oito modelos de aceitação estudados anteriormente, incluindo TPB, TRA e TAM, para explicar o impacto da adoção da tecnologia nas organizações (Figura 5). A teoria se baseia em quatro construtos fundamentais, sendo um deles a Expectativa de Desempenho, que apresentou relação positiva com o uso da tecnologia.

Figura 5 - UTAUT

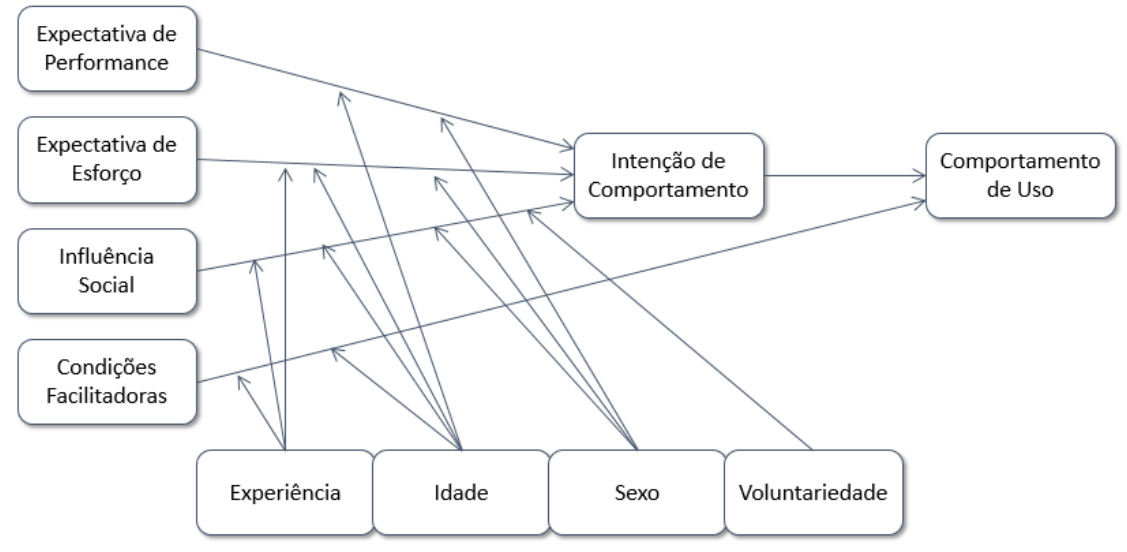

Fonte: Adaptado de Venkatesh et al. (2003) 
De acordo com Visentini, Bobsin e Rech (2008), ao menos outros quatro modelos consideram a Expectativa de Desempenho como construto relevante: MM - Modelo Motivacional (MM) (VALLERAND, 1997); MPCU - Modelo de Utilização do PC (THOMPSON; HIGGINS; HOWELL, 1991); IDT - Teoria da Difusão da Inovação (ROGERS, 1995) e SCT Teoria Social Cognitiva (BANDURA, 1986).

Quando se trata de sistemas de informação, a expectativa do usuário é definida como o conjunto de crenças mantidas pelos usuários associadas ao seu desempenho com a utilização do sistema (SZAJNA; SCAMELL, 1993). O estudo de Tse e Wilton (1988) encontrou suporte à influência das expectativas previstas sobre a satisfação e o desempenho em determinado produto existente.

Outros estudos sobre sistemas especialistas (programas destinados a solucionar problemas em campos específicos de conhecimento) verificaram uma forte correlação positiva entre expectativa, melhor desempenho e níveis de satisfação (MAHMOOD et al., 2000; YOON; GUIMARAES; O'NEAL, 1995). No âmbito do entretenimento, a expectativa de desempenho se apresenta quando novos desafios são trazidos e o indivíduo não passa de nível enquanto não vencer o atual, é preciso concluir toda a missão para que se possa avançar para outra fase impondo-se a busca por melhor desempenho (AIRES; PILATTI, 2016).

\section{METODOLOGIA}

Esta é uma pesquisa quantitativa, de natureza causal, cuja coleta de dados foi realizada por meio de questionário estruturado e autoadministrado (GIL, 2008). O questionário foi disponibilizado eletronicamente a partir do site https://pt.surveymonkey.com. Pelo fato de jogos on-line utilizarem como plataforma a Internet, cuja característica natural é a dispersão física, o convite para a resposta a essa pesquisa foi distribuído aleatoriamente e solicitado a cada respondente que a adicionasse nos fóruns de jogos on-line que participava.

\subsection{Método de pesquisa}

Quanto aos fins, essa pesquisa é considerada como explicativa, já que analisa as causas da intenção de jogar identificando fatores que determinam ou contribuem para a ocorrência dos fenômenos e aprofundando o conhecimento da realidade (GIL, 2008).

A população da pesquisa é estimada em 43 milhões de respondentes, de acordo com informação atualizada que quantifica em 102 milhões os internautas no Brasil (PORTAL BRASIL, 2016) e a pesquisa que revela que, no Brasil, $69 \%$ dos internautas também jogam games e desses, $61 \%$ jogam on-line (UOL, 2016). A amostra é intencional, já que foi escolhido intencionalmente um grupo de elementos (MARTINS; THEÓPHILO, 2009) e composta de 186 respondentes. Os dados foram coletados por meio de questionário estruturado utilizando escala Likert com sete opções de resposta variando entre 1 (Discordo Totalmente) até 7 (Concordo Totalmente).

Para a análise dos resultados foi utilizada a modelagem de equações estruturais (MEE), considerada como padrão em pesquisas de administração, quando se deseja analisar relações de causa e efeito entre construtos (HAIR; RINGLE; SARSTEDT, 2011). Empregou-se modelagem de equações estruturais, com mínimos quadrados parciais (MEE$M Q P)$, já que o objetivo da pesquisa é explicar a variância das variáveis dependentes (predição dos construtos).

Quando se utiliza MEE-MQP, os resultados devem ser avaliados em duas etapas: preliminarmente, analisa-se o modelo externo (de mensuração) que exibe as relações entre construtos e indicadores e, posteriormente, o modelo interno (estrutural), que exibe as relações (caminhos) entre os construtos. O modelo externo deve ser avaliado, inicialmente, pela verificação da confiabilidade dada pelo grau de isenção de erros aleatórios que a medida apresenta e, em seguida, por sua validade - grau em que a operacionalização do construto foi adequadamente realizada. Para verificarmos a confiabilidade foram utilizados os indicadores Alfa de Cronbach, Confiabilidade Composta (CC) e Variância Média Extraída (VME).

Em seguida, deve-se analisar a validade discriminante através do exame das cargas cruzadas dos indicadores, que é geralmente considerada mais liberal para estabelecer a validade discriminante. Uma abordagem mais conservadora consiste no critério de Fornell-Larcker, que compara a raiz quadrada das VME com as correlações entre variáveis (HAIR et al., 2014). Deve-se analisar a existência de multicolinearidade, por meio do fator de inflação de variância (VIF), um valor VIF de cinco, ou maior, indica um potencial problema de colinearidade (HAIR et al., 2014).

Seguimos para a avaliação dos coeficientes de determinação de Pearson $\left(R^{2}\right)$, medida da exatidão preditiva do modelo, calculado como a correlação quadrática entre os valores de um específico construto endógeno e os 
valores preditos. Hair et al (2012) exemplificam $\mathrm{R}^{2}$ de $0,75,0,50$ ou 0,25 como variável substancial, moderada ou fraca, respectivamente.

Foram verificados Relevância ou Validade Preditiva $\left(Q^{2}\right)$, ou indicador de Stone-Geisser, e Tamanho do efeito $\left(f^{2}\right)$, ou Indicador de Cohen. O Tamanho do Efeito $\left(\mathrm{f}^{2}\right)$ é a variação do valor $\mathrm{R}^{2}$ testado quando um construto exógeno específico é omitido do modelo e é avaliado se o impacto desse construto omitido é substantivo sobre o construto endógeno.

\subsection{Objetivos e modelo}

O objetivo central deste trabalho é analisar a importância da expectativa de desempenho na intenção de jogar on-line reforçando sua importância quando relacionada ao entretenimento.

Para responder a essa questão de pesquisa, utiliza-se o modelo da Figura 6, cuja construção foi baseada no conjunto de teorias que são básicas na formulação desse tema que, por sua vez, se sustentam nos seguintes construtos fundamentais:
1) Interação homem-computador
2) Interação social
3) Experiência de envolvimento
4) Percepção de prazer
5) Expectativa de desempenho
6) Atitude
7) Norma subjetiva
8) Percepção de controle comportamental
9) Intenção
10) Comportamento

Como objetivos intermediários, podemos relacionar:

A) Validar a consistência exploratória do modelo de adoção de tecnologia TPB no que tange a jogos online, corroborando o estudo de Lee (2009) e mapeando e identificando pontos a serem observados com maior atenção na fase de estudo de projetos desse tipo.

B) Validar a importância ressaltada por Lee (2009) para o construto Experiência de Envolvimento na intenção de jogar.

Figura 6 - Modelo de adoção proposto

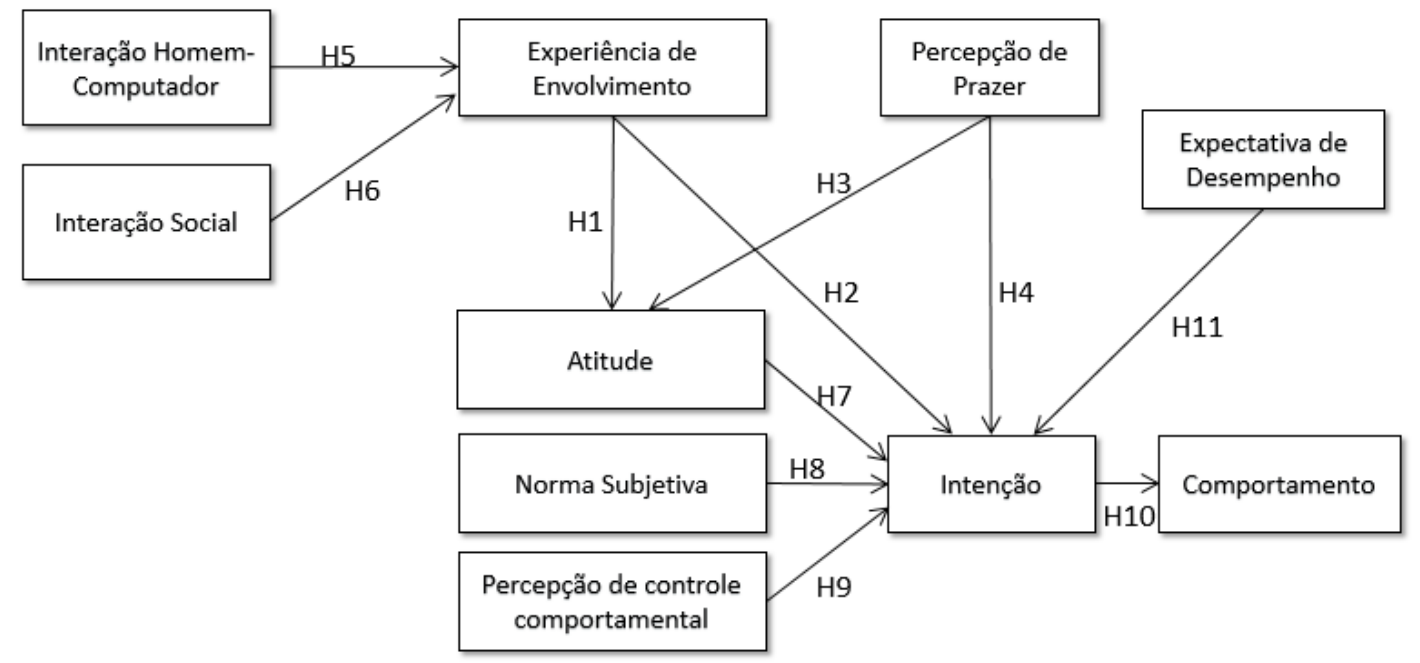

Fonte: Elaboração Própria (2017).

As seguintes hipóteses oriundas das teorias e reproduzidas no estudo de Lee (2009) são considerados neste estudo:

- $\quad H 1$ - Experiência de Envolvimento é positivamente relacionada à Atitude de jogar on-line.

- $\quad$ H2 - Experiência de Envolvimento é positivamente relacionada à Intenção de jogar on-line. 
A Experiência de Envolvimento é definida como "a sensação holística que as pessoas sentem quando agem com total envolvimento".

- H3 - Percepção de Prazer é positivamente relacionada à Atitude de jogar on-line.

- H4 - Percepção de Prazer é positivamente relacionada à Intenção de jogar on-line.

- Usar uma tecnologia de forma divertida e agradável motiva seus usuários a adotá-la.

- H5 - Interação Homem-Computador é positivamente relacionada à Experiência de Envolvimento ao jogar on-line.

A interface homem-computador é definida como o ponto de contato entre a aplicação e o usuário final. A comunicação interativa entre usuários e jogos on-line ocorre através de interfaces de hardware e software.

- $\quad H 6$ - Interação Social é positivamente relacionada à Experiência de Envolvimento ao jogar on-line.

- $\quad$ H7 - Atitude é positivamente relacionada à Intenção de jogar on-line.

A atitude é definida como a avaliação geral de um indivíduo ao realizar um comportamento e afeta a intenção comportamental dos usuários (AJZEN, 1991).

- H8 - Norma Subjetiva é positivamente relacionada à Intenção de jogar on-line

- H9 - Percepção de Controle Comportamental é positivamente relacionada à Intenção de jogar online.

Percepção de Controle Comportamental refere-se à "percepção das pessoas da facilidade ou dificuldade em realizar o comportamento de interesse" (AJZEN, 1991).

- H10 Intenção de Jogar on-line é positivamente relacionada à Comportamento.

Além dessas já explicitadas por Lee (2009) em seu estudo, a seguinte hipótese foi incorporada:

- H11 - A Expectativa de Desempenho afeta positivamente à Intenção de jogar on-line.

De acordo com Davis, Bagozzi e Warshaw (1989), a expectativa de desempenho é o grau que uma pessoa acredita que utilizar um determinado sistema, aqui considerado como um jogo on-line, permitiria reforçar o seu desempenho, aqui considerado o desempenho no ambiente do próprio jogo.

\subsection{Pesquisa de campo}

A pesquisa quantitativa utiliza a escala testada e validada por Lee (2009) para avaliar empiricamente o modelo proposto, incluindo o construto Expectativa de Desempenho em jogos on-line. Os itens do questionário foram adaptados a partir de modelos de aceitação de tecnologia para o contexto de jogos on-line, representando variáveis dependentes e independentes a serem testadas no estudo atual. Todos os itens foram medidos em uma escala Likert de sete pontos.

\section{RESULTADOS E DISCUSSÃO}

A amostra é composta por 186 respondentes, sendo que $3 / 4$ do sexo masculino e $1 / 4$ do sexo feminino. A idade está distribuída como apresentado na Figura 7. A experiência prévia é apresentada na Figura 8.

Figura 7 - Distribuição por idade

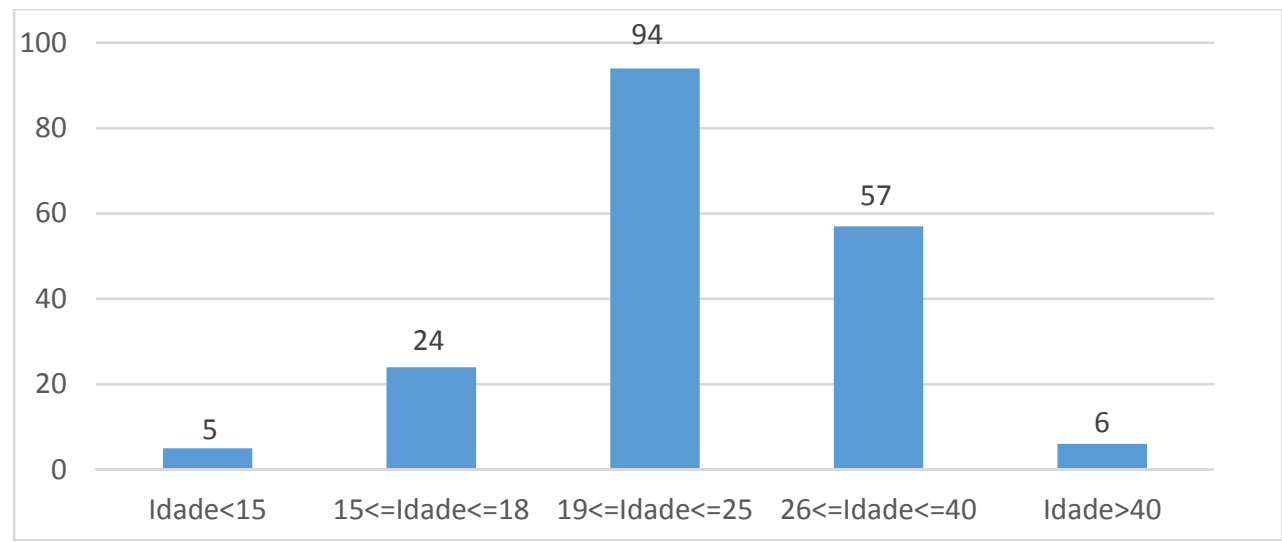

Fonte: Elaboração Própria (2017). 
Figura 8 - Experiência prévia

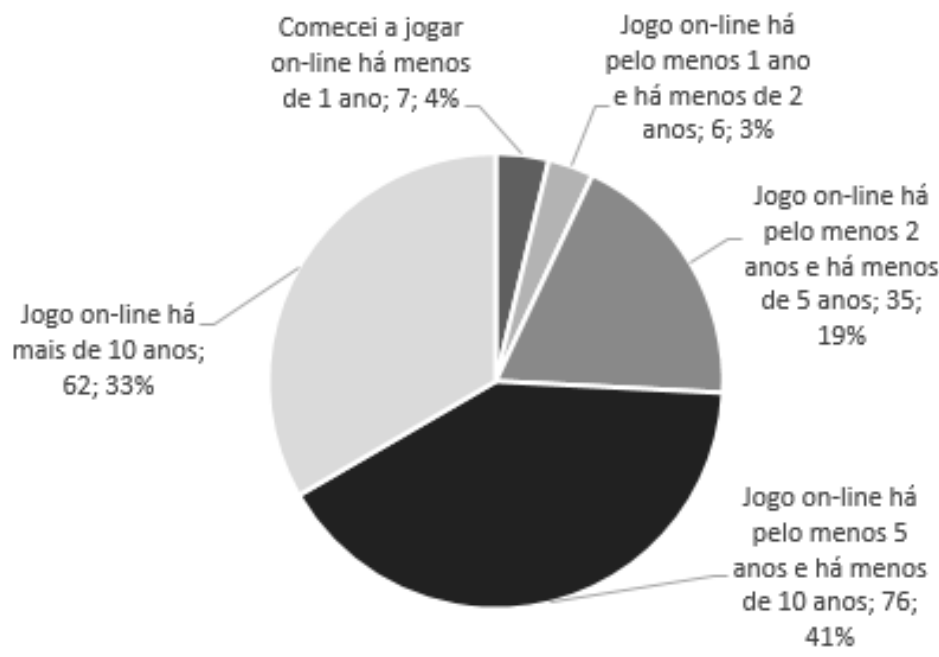

Fonte: Elaboração Própria (2017).

Inicialmente, foi verificada a confiabilidade pelo grau de isenção de erros aleatórios que a medida apresenta e, em seguida, por sua validade - grau em que a operacionalização do construto foi adequadamente realizada. Para a Confiabilidade Composta, valores abaixo de 0,60 e acima de 0,95 não são desejáveis (HAIR et al., 2009). Todos os valores encontrados situam-se na faixa dos valores desejados (Tabela 2). Há consenso de que o valor crítico inferior para o alfa de Cronbach é 0,70 (HAIR et al., 2009), nesse estudo, os construtos Percepção de Controle Comportamental e Experiência de Envolvimento apresentaram valores abaixo do valor mínimo, indicando carência de consistência interna (Tabela 2).

Para a variância média extraída, o valor de 0,50 é o valor mínimo considerado aceitável (HAIR et al., 2014). Nesse estudo os construtos Percepção de Controle Comportamental e Experiência de Envolvimento apresentaram valores abaixo do valor mínimo (Tabela 2), o que indica que explicam menos do que a média de variância de seus indicadores, não sendo, portanto, considerados satisfatórios.

Tabela 2 - Alpha de Cronbach, CC e VME

\begin{tabular}{lccc} 
& $\begin{array}{c}\text { Alpha de } \\
\text { Cronbach }\end{array}$ & $\begin{array}{c}\text { Confiabilidade } \\
\text { Composta }\end{array}$ & $\begin{array}{c}\text { Variância } \\
\text { Média Extraída }\end{array}$ \\
\hline \hline Interação Social & 0.777 & 0.857 & 0.600 \\
Interação Homem Computador & 0.900 & 0.937 & 0.833 \\
Atitude & 0.871 & 0.922 & 0.797 \\
Norma Subjetiva & 0.845 & 0.907 & 0.765 \\
Percepção de Controle Comportamental & 0.355 & 0.683 & 0.458 \\
Experiência de Envolvimento & 0.656 & 0.784 & 0.422 \\
Percepção de Prazer & 0.875 & 0.923 & 0.800 \\
Expectativa de Desempenho & 0.885 & 0.929 & 0.814 \\
Intenção de Jogar & 0.880 & 0.926 & 0.807 \\
Comportamento & 0.764 & 0.894 & 0.809
\end{tabular}

Fonte: Elaboração Própria (2017).

Analisando-se os valores P encontrados, os construtos Percepção de Controle Comportamental e Experiência de Envolvimento novamente apresentaram valores abaixo do valor mínimo reforçando a falta de relevância estatística já identificada nas análises anteriores, somado ao construto Interação Homem Computador que também não apresentou relevância estatística. Os outros construtos apresentam valores relevantes (Tabela 3). 
Tabela 3 - Valores P

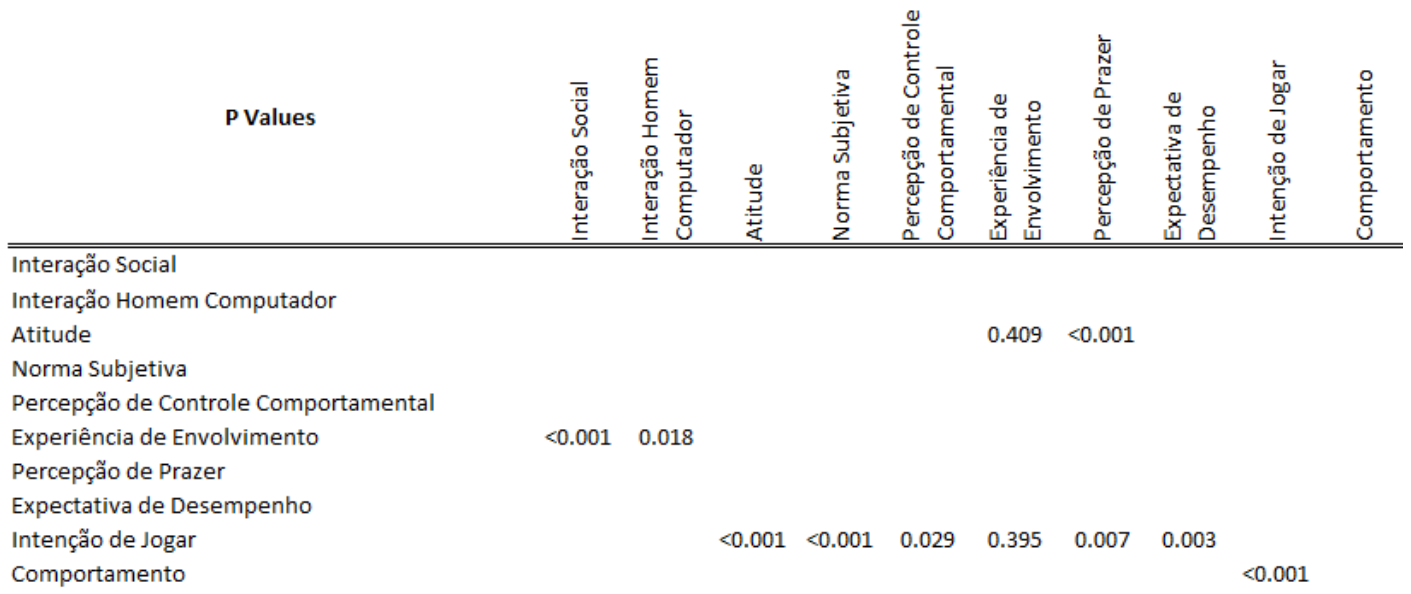

Fonte: Elaboração Própria (2017).

$\mathrm{Na}$ análise da validade discriminante foi feito o exame das cargas cruzadas dos indicadores conforme Tabela

4.

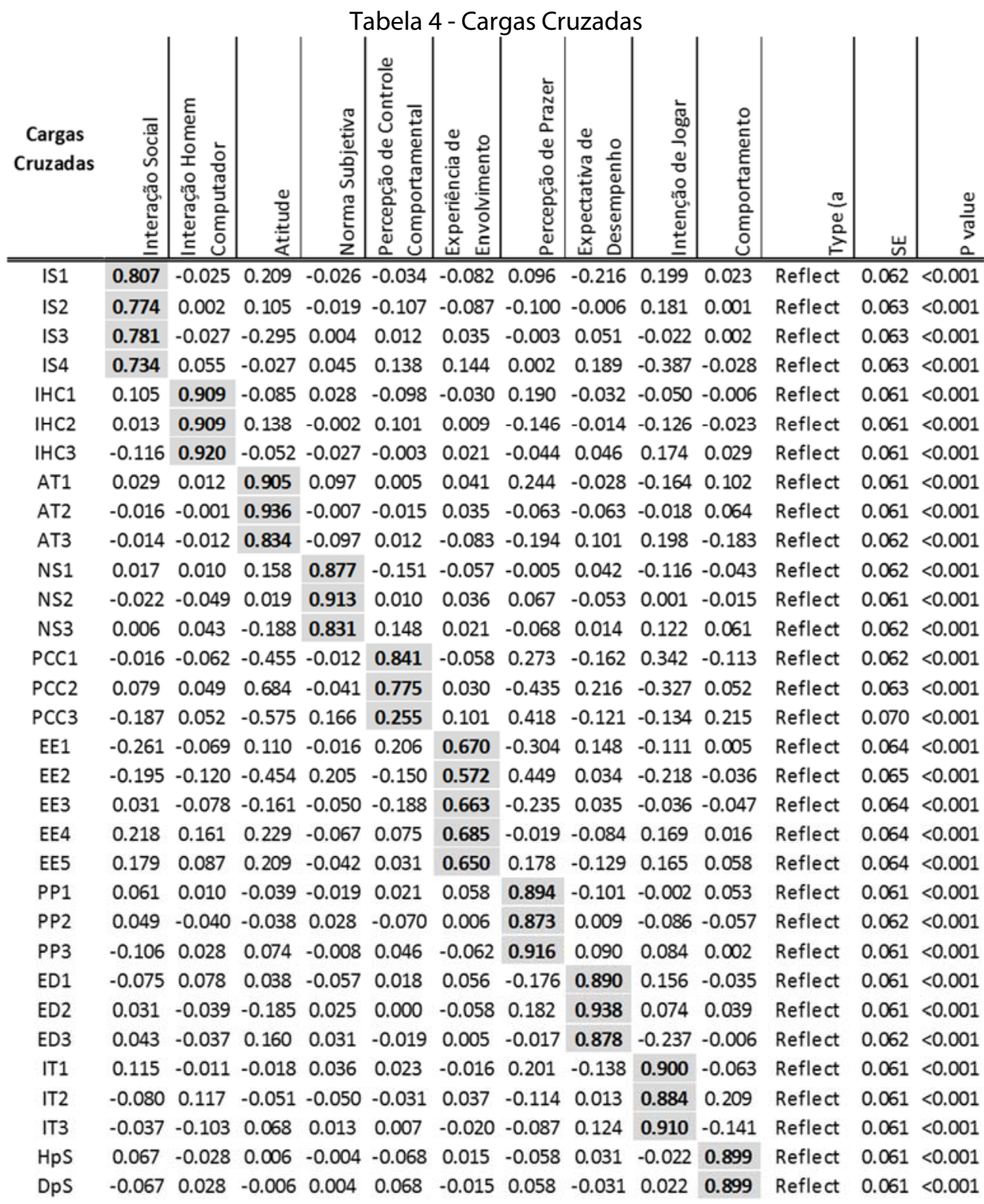

Fonte: Elaboração Própria (2017). 
Na Tabela 5, podemos observar o tamanho do efeito (f2). Os parâmetros para avaliação de f2 são 0,02, 0,15 e 0,35, representando efeitos pequeno, médio e grande da variável latente exógena, respectivamente (HAIR et al., 2014). Os coeficientes de caminho do modelo estrutural ( $\beta$ ) representam relações hipotéticas entre os construtos. Coeficientes de caminho têm valores padronizados entre -1 e 1, sendo que os valores próximos a \pm 1 representam relação forte entre construtos. Quanto mais próximos de zero estiverem os coeficientes, mais fraca a relação (Tabela 5).

Tabela 5 - Tamanho do efeito e coeficiente de caminho

\begin{tabular}{lcc}
\multicolumn{1}{c}{ Caminho } & f2 & $\boldsymbol{\beta}$ \\
\hline \hline Experiência de Envolvimento $\rightarrow$ Atitude & 0.011 & 0.017 \\
Percepção de Prazer $\rightarrow$ Atitude & 0.672 & 0.813 \\
Interação Social $\rightarrow$ Experiência de Envolvimento & 0.147 & 0.344 \\
Interação Homem Computador $\rightarrow$ Experiência de Envolvimento & 0.051 & 0.151 \\
Atitude $\rightarrow$ Intenção de Jogar & 0.357 & 0.489 \\
Norma Subjetiva $\rightarrow$ Intenção de Jogar & 0.090 & 0.232 \\
Percepção de Controle Comportamental $\rightarrow$ Intenção de Jogar & 0.059 & 0.136 \\
Experiência de Envolvimento $\rightarrow$ Intenção de Jogar & 0.009 & -0.019 \\
Percepção de Prazer $\rightarrow$ Intenção de Jogar & 0.115 & 0.176 \\
Expectativa de Desempenho $\rightarrow$ Intenção de Jogar & 0.113 & 0.193 \\
Intenção de Jogar $\rightarrow$ Comportamento & 0,242 & 0.491
\end{tabular}

Fonte: Elaboração Própria (2017).

Por fim, avaliou-se a validade discriminante, com base no critério de Fornell-Larcker, por ser uma abordagem mais conservadora. A raiz quadrada de cada variância média extraída (VME) deve ser superior a sua maior correlação com qualquer outro construto, o que se confirma na Tabela 6.

Tabela 6 - Correlações entre construtos e VME

\begin{tabular}{|c|c|c|c|c|c|c|c|c|c|c|}
\hline $\begin{array}{l}\text { Correlações entre os } \\
\text { construtos com a } \\
\text { raiz quadrada da VME }\end{array}$ & 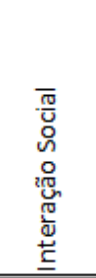 & 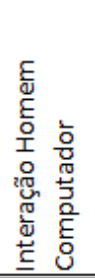 & 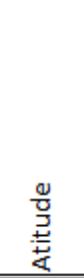 & 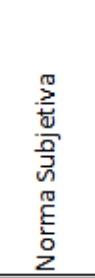 & 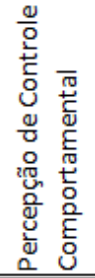 & 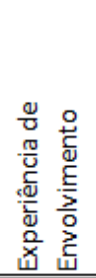 & 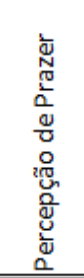 & 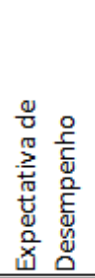 & 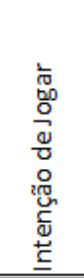 & 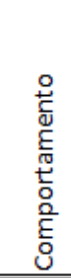 \\
\hline Interação Social & 0.774 & & & & & & & & & \\
\hline Interação Homem Computador & 0.320 & 0.913 & & & & & & & & \\
\hline Atitude & 0.513 & 0.447 & 0.893 & & & & & & & \\
\hline Norma Subjetiva & 0.140 & 0.182 & 0.256 & 0.874 & & & & & & \\
\hline Percepção de Controle Comportamental & 0.206 & 0.309 & 0.407 & 0.191 & 0.677 & & & & & \\
\hline Experiência de Envolvimento & 0.387 & 0.266 & 0.425 & 0.105 & 0.047 & 0.649 & & & & \\
\hline Percepção de Prazer & 0.484 & 0.468 & 0.799 & 0.178 & 0.464 & 0.469 & 0.895 & & & \\
\hline Expectativa de Desempenho & 0.476 & 0.397 & 0.616 & 0.162 & 0.416 & 0.367 & 0.652 & 0.902 & & \\
\hline Intenção de Jogar & 0.519 & 0.438 & 0.727 & 0.389 & 0.328 & 0.356 & 0.650 & 0.566 & 0.898 & \\
\hline Comportamento & 0.297 & 0.149 & 0.426 & 0.055 & 0.106 & 0.213 & 0.274 & 0.309 & 0.478 & 0.899 \\
\hline
\end{tabular}

Fonte: Elaboração Própria (2017).

A análise dos dados do modelo interno apresenta consistência entre os índices avaliados e também com as análises do modelo externo. O tamanho do efeito e o coeficiente de caminho apresentaram índices maiores para o construto Percepção de Prazer, seguido por Atitude, Expectativa de Desempenho e Interação Social. Os construtos Experiência de Envolvimento, Interação Homem-Computador, Percepção de Controle Comportamental apresentaram resultado insatisfatório, o que também se repetiu na análise do modelo externo. Esses resultados revelam o seguinte impacto nas hipóteses avaliadas (Quadro 1). 
Quadro 1 - Avaliação das hipóteses

\begin{tabular}{|l|c|}
\hline \multicolumn{1}{|c|}{ Hipótese } & Avaliação \\
\hline \hline $\begin{array}{l}\text { H1 Experiência de Envolvimento é positivamente relacionada à Atitude de jogar } \\
\text { on-line. }\end{array}$ & Rejeitada \\
\hline $\begin{array}{l}\text { H2 Experiência de Envolvimento é positivamente relacionada à Intenção de jogar } \\
\text { on-line. }\end{array}$ & Rejeitada \\
\hline H3 Percepção de Prazer é positivamente relacionada à Atitude de jogar on-line. & Confirmada \\
\hline H4 Percepção de Prazer é positivamente relacionada à Intenção de jogar on-line. & Confirmada \\
\hline $\begin{array}{l}\text { H5 Interação Homem-Computador é positivamente relacionada à Experiência de } \\
\text { Envolvimento ao jogar on-line. }\end{array}$ & Rejeitada \\
\hline $\begin{array}{c}\text { H6 Interação Social é positivamente relacionada à Experiência de Envolvimento } \\
\text { ao jogar on-line. }\end{array}$ & Confirmada \\
\hline H7 Atitude é positivamente relacionada à Intenção de jogar on-line. & Confirmada \\
\hline H8 Norma Subjetiva é positivamente relacionada à Intenção de jogar on-line. & Rejeitada \\
\hline $\begin{array}{l}\text { H9 Percepção de Controle Comportamental é positivamente relacionada à } \\
\text { Intenção de jogar on-line. }\end{array}$ & Rejeitada \\
\hline H10 Intenção de Jogar on-line é positivamente relacionada à Comportamento. & Confirmada \\
\hline H11 Expectativa de Desempenho afeta positivamente à Intenção de jogar on-line. & Confirmada \\
\hline
\end{tabular}

Fonte: Elaboração Própria (2017).

A partir da rejeição das hipóteses $\mathrm{H} 1$ e H2, o construto Experiência de Envolvimento deixa de ser relevante no modelo final. Como Interação Homem-Computador e Interação Social impactam a Intenção de Jogar somente a partir do construto Experiência de Envolvimento, estes também deixam de ser relevantes no modelo final.

O mesmo raciocínio se aplica aos construtos Norma Subjetiva e Percepção do Controle Comportamental cujas hipóteses $\mathrm{H} 8$ e $\mathrm{H} 9$, respectivamente, foram rejeitadas.

Dessa forma, o modelo final pode ser visto na Figura 9 onde a percepção de prazer influencia diretamente a atitude $(\mathrm{H} 3)$ e a intenção de jogar $(\mathrm{H} 4)$ que, por sua vez, sofre influência da expectativa de desempenho $(\mathrm{H} 11)$.

Figura 9 - Modelo Final

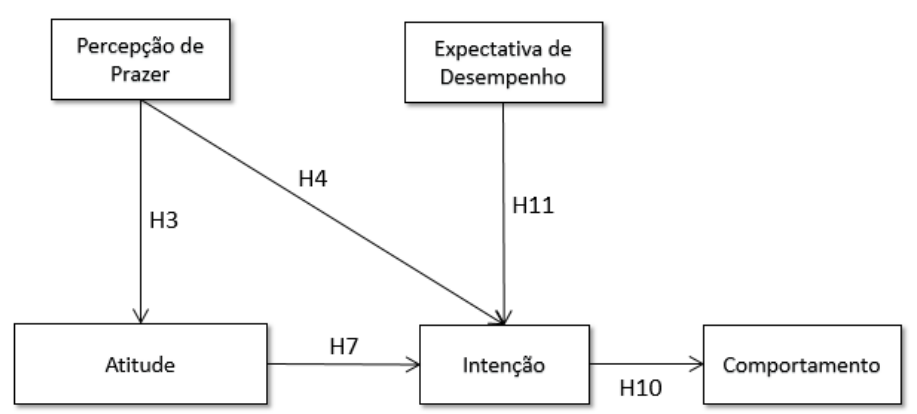

Fonte: Elaboração própria 


\section{CONCLUSÃO}

Este estudo respondeu positivamente ao seu objetivo principal: A expectativa de desempenho influencia a intenção de jogar on-line. Também respondeu aos seus objetivos intermediários:

I. Foi validada a consistência exploratória do modelo de adoção de tecnologia TPB no que tange a jogos on-line, corroborando o estudo de Lee (2009); foram mapeados e identificados pontos a serem observados com maior atenção na fase de estudo de projetos desse tipo, em especial, os construtos que não apresentaram relevância estatística;

II. Não foi encontrada relevância estatística que apoiasse a importância ressaltada por Lee (2009) para o construto Experiência de Envolvimento na intenção de jogar.

Para que fosse mantida estrita relação com os objetivos definidos, não foram aplicadas as ações de correção do modelo retirando os construtos sem relevância estatística. Esta linha de ação abre a possibilidade de novos estudos que complementem esta análise.

O fato da experiência de envolvimento ser fator chave para Lee e não ter apresentado relevância estatística, ao mesmo tempo que expectativa de desempenho se apresentou como fator relevante, pode demonstrar que jogar cada vez melhor é mais importante que a sensação holística preconizada por Lee (2009). Concluir toda a missão, passando por cada nível para que se possa avançar, obriga que a busca pelo melhor desempenho seja o fator principal.

Percepção de Prazer e Interação Social foram os dois construtos com mais relevância, isso denota uma maior influência de fatores voltados ao relacionamento interpessoal do que os fatores relacionados ao indivíduo como experiência de envolvimento e interação homem-computador, que tiveram suas hipóteses rejeitadas.

Expectativa de desempenho, apesar de estar relacionado ao desempenho do indivíduo, também pode ser considerado um fator de relacionamento já que o desempenho individual afeta o grupo e sua progressão como equipe. Querer evoluir individualmente e ser mais competitivo requer jogar por mais tempo.

O modelo final considerando apenas percepção de prazer e expectativa de desempenho condiz com o ambiente do entretenimento, foco central quando se joga on-line.

Para a academia, esse estudo contribui para aumentar a compreensão teórica dos fatores que influenciam os jogos on-line. No estudo prévio, Lee (2009) considera a experiência de envolvimento como fator-chave influenciador da intenção de jogar e desconsiderou a expectativa de desempenho, já que é um tema normalmente associado ao contexto empresarial. Entretanto, no entretenimento, há uma diferença substancial para o contexto empresarial, a importância das intenções individuais de melhora a cada jogo é fator que deve ser considerado pelos desenvolvedores de jogos on-line.

Esse estudo apresenta algumas limitações. Em primeiro lugar, o estudo foi feito a partir da distribuição de questionário pela Internet com a sugestão de que fosse publicado em fóruns, comuns em jogos on-line. Essa forma de coleta de respondentes pode reduzir a generalização já que tende a concentrar em poucos grupos. Pesquisas futuras devem procurar expandir a coleta de forma a determinar até que ponto os resultados aqui apresentados se aplicam de forma mais abrangente.

Em segundo lugar, esse estudo se baseia em uma fotografia retirada em um período de 30 dias de coleta de dados, o que determina o caráter transversal nessa pesquisa. Em estudo longitudinal, o modelo de pesquisa abrangeria diferentes períodos de tempo e as comparações seriam mais ricas, proporcionando mais insights sobre a adoção de jogos on-line.

Por último, a TPB está, em princípio, aberta à inclusão de construtos adicionais, conforme especifica seu autor. No entanto, qualquer inclusão deve ser feita com cautela, e somente após cuidadosa deliberação e exploração empírica (AJZEN, 1991). A inclusão do construto Expectativa de Desempenho se mostrou estatisticamente relevante e conceitualmente alinhada com o modelo, mas novas pesquisas devem procurar confirmar e discutir a abrangência dessa ampliação no modelo. 


\section{REFERÊNCIAS}

AIRES, J. P.; PILATTI, L. A. Aprendizagem significativa por meio do ensino adaptativo. Revista Espacios, v. 37, n. 29, p. 18, 2016.

AJZEN, I. The theory of planned behavior. Organizational Behavior and Human Decision Processes, v. 50, p. 179$211,1991$.

BAGATTOLI, L. S.; MÜLLER, G. C. K. Treinamento e desenvolvimento de pessoal : agregando valor às pessoas e à organização. Navus - Revista de Gestão e Tecnologia, v. 6, n. 2, p. 106-120, 2016.

BANDURA, A. Social foundations of thought and action: a social cognitive theory. Michigan: Prentice-Hall, 1986.

BOUZADA, M. A. C. O impacto da estratégia de centralização de estoques: uma análise em um laboratório de logística. REUNA Revista dos Programas de Mestrado do Centro Universitário UNA, v. 18, n. 3, p. 41-56, 2013.

CHINOMONA, R. Mobile Gaming Perceived Enjoyment and Ease of Play as Predictors of Student Attitude and Mobile Gaming Continuance Intention. Mediterranean Journal of Social Sciences MCSER Publishing, v. 4, n. 14, p. 237$247,2013$.

DAVIS, F. A Technology Acceptance Model for Empirically Testing New End-User Information Systems. [s.I.]: MIT - Massachusetts Institute of Technology, 1985.

DAVIS, F. D.; BAGOZZI, R. P.; WARSHAW, P. R. User Acceptance Of Computer Technology: A Comparison Of Two. Management Science, v. 35, n. 8, p. 982-1003, 1989.

FISHBEIN, M.; AJZEN, I. Belief, Attitude, Intention, and Behavior. Massachusetts: Addison-Wesley Pub., 1975.

GIL, A. C. Métodos e técnicas de pesquisa social. 6. ed. São Paulo: Atlas, 2008.

HAIR, J. F. et al. Análise Multivariada de Dados. 6. ed. Porto Alegre: Bookman Editora, 2009.

HAIR, J. F. et al. An assessment of the use of partial least squares structural equation modeling in marketing research. Journal of the Academy of Marketing Science, v. 40, n. 3, p. 414-433, 2012.

HAIR, J. F. J. et al. Partial least squares structural equation modeling (PLS-SEM). Sage Publisher, v. 26, n. 2, p. 106121, 2014.

HAIR, J. F.; RINGLE, C. M.; SARSTEDT, M. PLS-SEM: Indeed a Silver Bullet. Journal of Marketing Theory and Practice, v. 19, n. 2, p. 139-152, 2011.

HO, C. I.; CHANG, C. C.; LEE, S. S. Exploring Key Determinants of Gamer Behavior for Somatosensory Video Games: An Application of the Extended Technology Acceptance Model and Game Flow Theory. Global Journal of Emerging Trends in e-Business, Marketing and Consumer Psychology, v. 1, n. 3, p. 154-171, 2014.

HSU, C. L.; LU, H. P. Why do people play on-line games? An extended TAM with social influences and flow experience. Information \& Management, v. 41, p. 853-868, 2004.

KIM, S.; CHANG, M. Computer Games for the Math Achievement of Diverse Students. Journal of Educational Technology \& Society, v. 13, n. 3, p. 224-232, 2010.

LEE, M. C. Understanding the behavioural intention to play online games: An extension of the theory of planned behaviour. Online Information Review, v. 33, n. 5, p. 849-872, 2009.

LEE, Y. H.; LIN, H. "Gaming is my work": identity work in internet-hobbyist game workers. Work, Employment \& Society, v. 25, n. 3, p. 451-467, 2011.

MAHMOOD, M. et al. Variables affecting information technology end-user satisfaction: a meta-analysis of the empirical literature. International Journal of Human-Computer Studies, v. 52, n. 4, p. 751-771, 2000. 
MARANGUNIĆ, N.; GRANIĆ, A. Technology acceptance model: a literature review from 1986 to 2013. Universal Access in the Information Society, v. 14, n. 1, p. 81-95, 2015.

NEWZOO. 2016 Global Games Market Report. San Francisco: Newzoo, 2016. Disponível em: <https://newzoo.com/insights/articles/global-games-market-reaches-99-6-billion-2016-mobile-generating-37/>. Acesso em: 10 dez. 2016.

NOBRE, A. C. DOS S.; RAMOS, A. S. M.; NASCIMENTO, T. C. Adoção de Práticas de Gestão de Segurança da Informação: Um Estudo com Gestores Públicos. REUNA Revista dos Programas de Mestrado do Centro Universitário UNA, v. 16, n. 4, p. 95-113, 2011.

PILZ, C.; FERRAZ, F. Analysis of Business Intelligence (Bi) Tools Adoption in Brazilian Companies. International Journal of Scientific Knowledge, v. 3, n. 4, p. 1-9, 2013.

PORTAL BRASIL. Pesquisa revela que mais de 100 milhões de brasileiros acessam a internet. Disponível em: <http://www.brasil.gov.br/ciencia-e-tecnologia/2016/09/pesquisa-revela-que-mais-de-100-milhoes-de-brasileirosacessam-a-internet>. Acesso em: 4 dez. 2016.

ROGERS, E. M. Diffusion of innovations. 3rd ed. New York: The Free Press, 1995.

SZAJNA, B.; SCAMELL, R. W. The Effects of Information System User Expectations on Their Performance and Perceptions. MIS Quarterly: Management Information Systems, v. 17, n. 4, p. 493-516, 1993.

TAY, L. "Serious games" for the Aussie industry - Software - iTnews. Disponível em: <http://www.itnews.com.au/news/serious-games-for-the-aussie-industry-174083>. Acesso em: 4 dez. 2016.

THEÓPHILO, C. R.; MARTINS, G. de A. Metodologia da Investigação Científica para Ciências Sociais Aplicadas. 2. ed. São Paulo: Atlas, 2009.

THOMPSON, R. L.; HIGGINS, C. A.; HOWELL, J. M. Personal computing: Toward a conceptual model of utilization. MIS Quarterly: Management Information Systems, v. 15, n. 1, p. 124-143, 1991.

TSE, D. K.; WILTON, P. C. Models of consumer satisfaction formation: an extension. Journal of marketing research, v. 25, p. 204-212, May 1988.

UOL. No Brasil, 69\% dos usuários de Internet jogam games, aponta pesquisa. Disponível em: <http://jogos.uol.com.br/ultimas-noticias/2016/05/02/no-brasil-69-dos-usuarios-de-internet-jogam-games-apontapesquisa.htm>. Acesso em: 1 jan. 2016.

VALLERAND, R. J. Toward A Hierarchical Model of Intrinsic and Extrinsic Motivation. In: ZANNA, M. P. (Ed.). . Advances in Experimental Social Psychology. San Diego, CA: Academic Press, 1997. v. 29. p. 271-360.

VENKATESH, V. et al. User Acceptance of Information Technology: Toward a Unified View. MIS Quarterly: Management Information Systems, v. 27, n. 3, p. 425-478, 2003.

VENKATESH, V.; DAVIS, F. D. A Theoretical Extension of the Technology Acceptance Model: Four Longitudinal Field Studies. Management Science, v. 46, n. 2, p. 186-204, 2000.

VISENTINI, M. S.; BOBSIN, D.; RECH, I. Ampliando as considerações sobre o uso da Tecnologia: o estado da arte do UTAUT. Encontro da ANPAD, 32., 2008, Rio de Janeiro. Anais...Rio de Janeiro/RJ: ANPAD, 2008. p. 1-16.

WASKO, M. et al. Stepping into the internet: New ventures in virtual worlds. MIS Quarterly: Management Information Systems, v. 35, n. 3, p. 645-652, 2011.

XIAO, B.; BENBASAT, I. E-Commerce Product Recommendation Agents : Use, Characteristics, and Impact. MIS Quarterly: Management Information Systems, v. 31, n. 1, p. 137-209, 2007.

YOON, Y.; GUIMARAES, T.; O'NEAL, Q. Exploring the Factors Associated with Expert Systems Success. MIS Quarterly: Management Information Systems, v. 19, n. 1, p. 83-106, 1995. 